\title{
Evolution of Raft Structure during Creep Deformation of The Ni-Based Single-Crystal Superalloy TMS-138
}

Nobuyasu Tsuno ${ }^{1, a}$, Akihiro Sato, b , Katsushi Tanaka ${ }^{2, c}$ and Haruyuki Inui ${ }^{2, d}$

${ }^{1} \mathrm{IHI}$ Corporation, 1 Shin-nakahara-cho, Isogo-ku, Yokohama, Japan

${ }^{2}$ Kyoto University, Yoshidahon-machi, Sakyo-ku, Kyoto, Japan

anobuyasu_tsuno@ihi.co.jp, baki_sato@ihi.co.jp, ${ }^{c k}$ atsushi-tanaka@mtl.kyoto-u.ac.jp,

dharuyuki.inui@materials.mbox.media.kyoto-u.ac.jp

Key words: Ni-based superalloy, Single crystal, Raft, Fourier analysis, Creep

\begin{abstract}
The evolution of the raft structure in the Ni-based single-crystal superalloy TMS-138 crept at $1100{ }^{\circ} \mathrm{C}$ and $137 \mathrm{MPa}$ has been investigated quantitatively by the Fourier analysis of scanning electron microscope (SEM) images. This method of analysis quantifies the extent of tilting of the $\gamma / \gamma^{\prime}$ interface during creep deformation. The analysis is carried out on two different crystallographic planes, (100) and (110), on which SEM observations of deformation microstructures are made, to see if there is any difference in the results of analysis depending on observation planes. The tilt angle of the $\gamma / \gamma^{\prime}$ interface, which is deduced by the Fourier analysis, increases with the accumulation of creep strain. The rate of the increase in the tilt angle is significantly large in the tertiary creep stage. The result of analysis does not depend significantly on crystallographic planes on which SEM observations of microstructures are made.
\end{abstract}

\section{Introduction}

The high temperature creep strength of Ni-based single-crystal superalloys is known to be closely related to the microstructure. When superalloys are subjected to creep deformation along the [001] direction in tension at high temperatures, $\gamma^{\prime}$ precipitates are coarsened preferentially in the (001) plane to form a lamellar structure that is usually called a 'raft' structure [1-5]. However, after the formation of the raft structure parallel to (001), the $\gamma / \gamma^{\prime}$ interface in the raft structure gradually tilts from the (001) plane so as to form a wavy lamellar interface with the wave amplitude increasing gradually as creep deformation proceeds [6-9]. Epishin et al. [9] characterized the evolution of the microstructure of the superalloy SRR99 during creep at $980{ }^{\circ} \mathrm{C}$ and $200 \mathrm{MPa}$ by Fourier analysis of scanning electron microscope (SEM) images on longitudinal (100) planes and successfully correlated the changes in microstructure with the accumulated creep strain. However, it is not obvious at present whether or not the method used by Epishin et al. [9] in characterizing the microstructure evolution of a particular superalloy during creep is applicable also to other superalloys crept under different temperatures and applied stresses. In the present study, we investigate the microstructure evolution of a different superalloy, TMS-138 during creep at a higher temperature, $1100{ }^{\circ} \mathrm{C}$ at $137 \mathrm{MPa}$ by Fourier analysis of SEM images to see the feasibility of their method. 


\section{Experimental procedure}

Specimens used were a Ni-based single-crystal superalloy TMS-138 with a chemical composition of Ni-5.8Co-3.2Cr-2.8Mo-5.9W-5.9Al-5.6Ta-0.1Hf-5.0Re-2.0Ru (in weight \%). The single- crystal superalloy in the fully heat-treated condition was provided by IHI casting Co. Ltd. The alloy contains cuboidal $\gamma^{\prime}$ precipitates with a mean size of about $400 \mathrm{~nm}$ with the volume fraction of about $70 \%$. Tensile creep tests were performed at $1100{ }^{\circ} \mathrm{C}$ and $137 \mathrm{MPa}$ along the [001] direction to several different accumulated strain levels. Thin plates were cut from crept specimens parallel not only to longitudinal (100) planes but also to longitudinal (110) planes to observe the microstructure evolution by SEM. These plates were mechanically polished and then etched in a $\gamma^{\prime}$ dissolving agent prior to SEM observations. Changes in the morphology of $\gamma / \gamma^{\prime}$ two-phase microstructures were characterized by Fourier analysis of SEM images with the use of a fast algorithm to get the amplitude spectra, as described by Epishin et al. [9].

\section{Results and discussion}

Creep strains of the superalloy TMS-138 crept at $1100{ }^{\circ} \mathrm{C}$ and $137 \mathrm{MPa}$ are plotted in Fig. 1 as a function of deformation time. The deformation time in Fig. 1 is normalized to the rupture time so as to express the life consumption ratio. The creep curve in Fig. 1 is a typical curve for creep deformation at high temperature and low stress, since it contains a short primary creep, followed by a long secondary creep and then by a rapidly accelerating tertiary creep [10-14]. SEM microstructures on longitudinal (110) planes and the corresponding FT spectra are depicted in Fig. 2 for different life consumption ratios. At a life consumption ratio of 0.03 , the crept specimen maintains a cuboidal microstructure (Fig. 2(a)), exhibiting four satellite spots around the origin in the FT spectrum (Fig. 2(f)). The distance between two satellite spots in the vertical direction is longer than that between two spots in the horizontal direction. This arises from the fact that the average distance between cuboidal precipitates is shorter in the vertical direction than in the horizontal direction in the (110) plane. When the life consumption ratio increases to 0.1 , the number

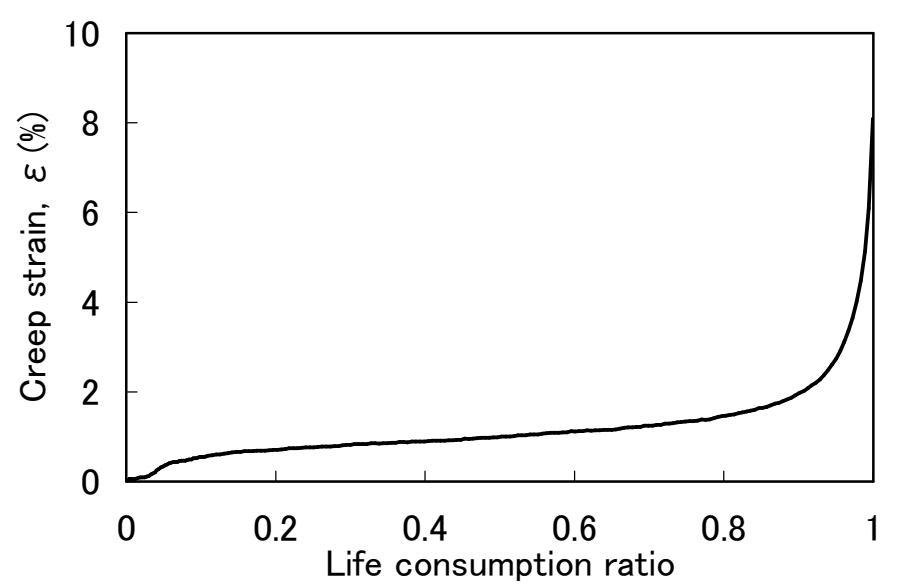

Fig. 1. Creep strain versus time curve obtained for a superalloy TMS- $138 \mathrm{crept}$ at $1100^{\circ} \mathrm{C}$ and $137 \mathrm{MPa}$. The creep time is normalized to the rupture time so as to express the life consumption.

of satellite spots is decreased to two in the vertical direction (Fig. 2(g)) because of the formation of the raft structure (Fig. 2(b)). Since the angular position of the satellite spot relative to the origin 
gives the mean direction perpendicular to the relevant $\gamma / \gamma^{\prime}$ interface, the angular width of the satellite spot represents an average tilt angle of the interfaces from the (001) plane in both positive and negative senses in the tilt angle. The tilt angle of the $\gamma / \gamma^{\prime}$ interface at the life consumption ratio
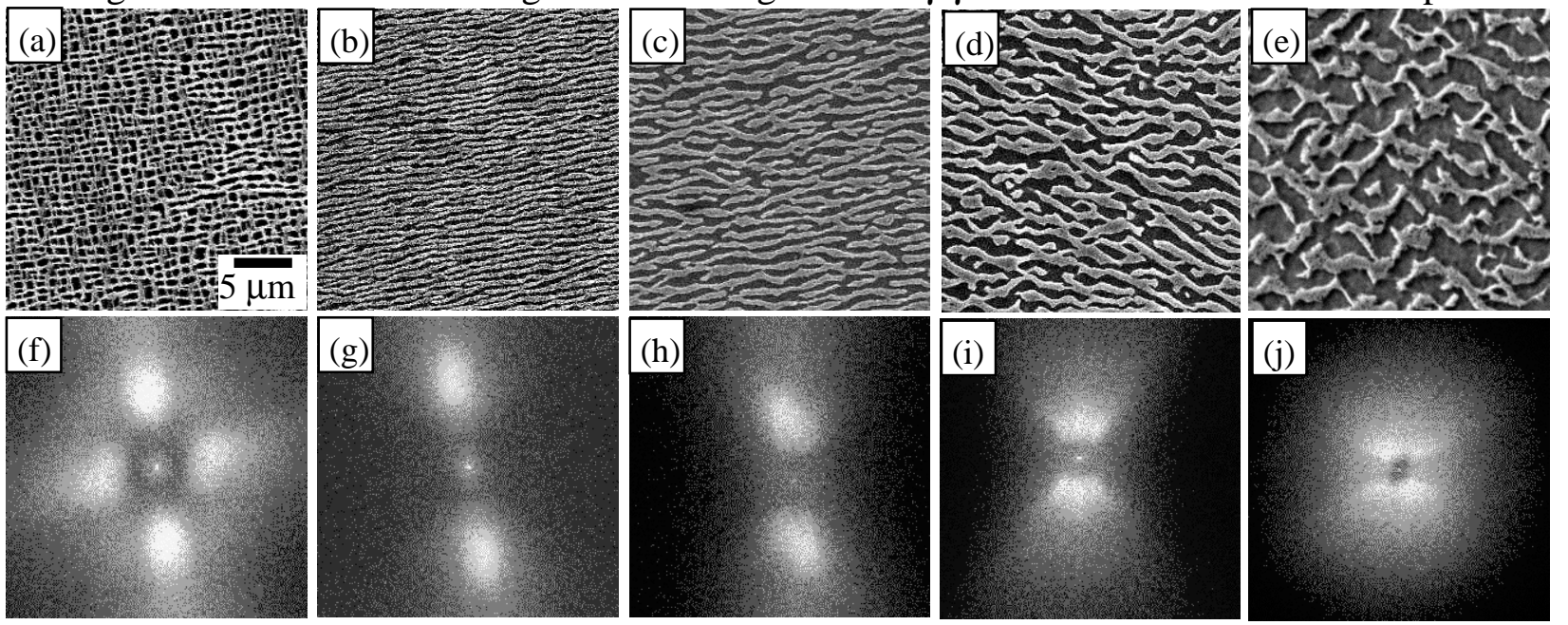

Fig. 2. (a)-(e) SEM microstructures on longitudinal (110) planes and (f)-(j) the corresponding FT spectra obtained at different life consumption ratios of (a), (f) 0.03, (b), (g) 0.1, (c), (h) 0.5, (d),(i) 0.95 and (e), (j) 1 .
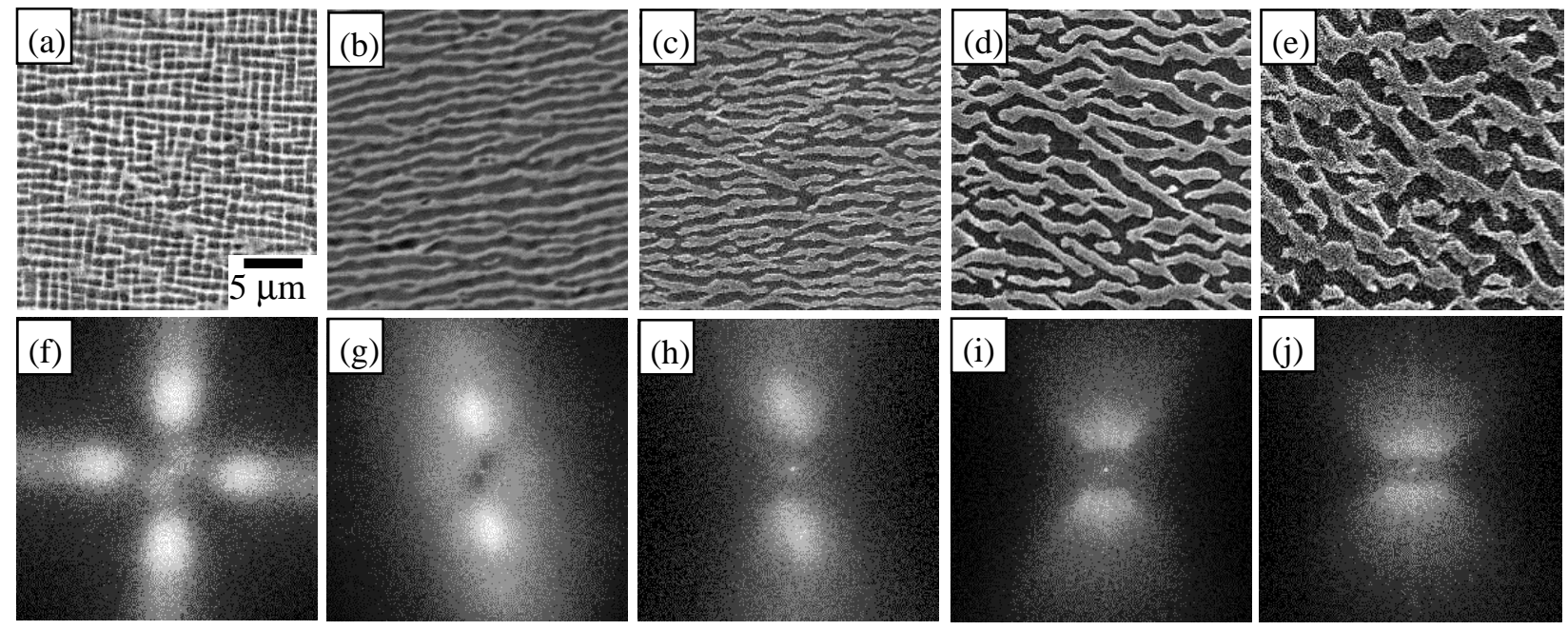

Fig. 3. (a)-(e) SEM microstructures on longitudinal (100) planes and (f)-(j) the corresponding FT spectra obtained at different life consumption ratios of (a), (f) 0.03, (b), (g) 0.1, (c), (h) 0.5, (d),

(i) 0.95 and (e), (j) 1 .

of 0.1 is thus determined to be $20^{\circ}$ from Fig. 2(g). As creep deformation proceeds, the raft structure coarsens and regions where the $\gamma / \gamma^{\prime}$ interface tilts from the (001) plane increase. Accordingly, the distance between the two satellite spots in the vertical direction decreases and their shapes become gradually obscured. Similar results are obtained also for observations on longitudinal (100) planes, as shown in Fig. 3.

The variation in the tilt angle of the $\gamma / \gamma^{\prime}$ interface with the life consumption ratio similarly determined for other crept specimens on both (100) and (110) planes is illustrated in Fig. 4. In the primary creep stage, the tilt angle of the $\gamma / \gamma^{\prime}$ interface increases with the accumulation of creep strain. The same trend is observed on both (100) and (110) planes, although the value of the tilt 
angle seems to be a little smaller on (110) planes than on (100) planes. In the secondary creep stage, the tilt angle further increases with the accumulation of creep strain, although the rate of the increase in the tilt angle with the creep strain is considerably decreased when compared to

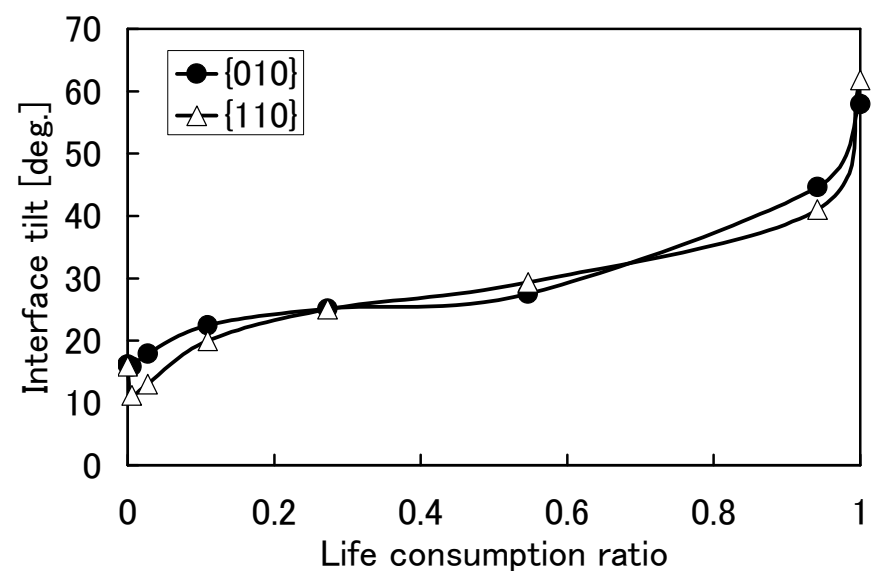

Fig. 4. Variation of the tilt angle of the $\gamma / \gamma^{\prime}$ interface deduced from observations on (100) and (110) planes with the life consumption ratios.

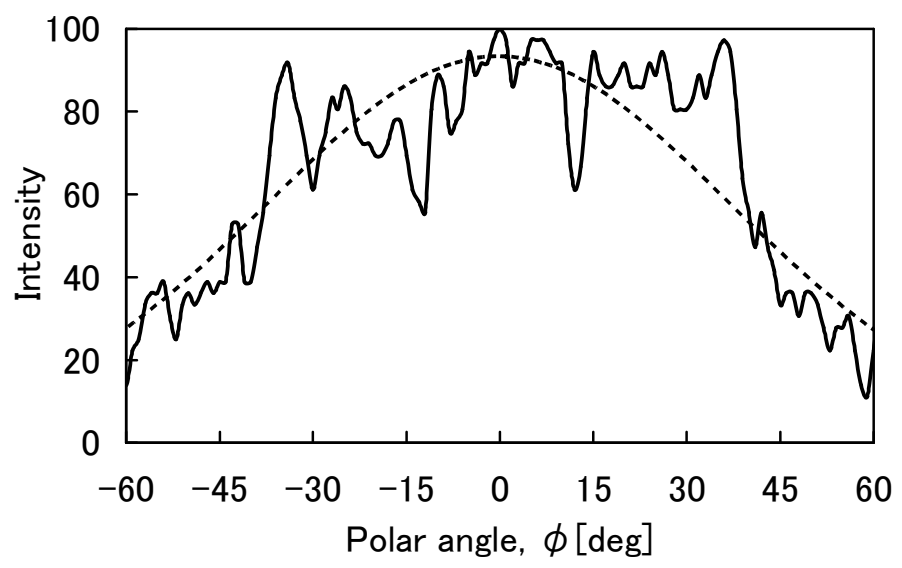

Fig. 5 Angular distribution profile of the FT spectrum obtained on (110) planes at the life consumption ratio of 0.95 .

that in the primary creep stage. The values of the tilt angle obtained on (100) planes are virtually identical with those obtained on (110) planes in this creep stage. In the tertiary creep stage, the tilt angle increases very rapidly with the accumulated creep strain regardless of the observation planes. The large scatter in the obtained tilt angles is due to the heterogeneity in the microstructure prior to rapture. These indicate that the microstructure evolution accompanied by the formation of the raft structure and the tilting of the $\gamma / \gamma^{\prime}$ interface in the raft structure during creep deformation of superalloys can be characterized by the Fourier transformation method without paying any special attention to the observation plane. Of significance to note is that the shape of the tilt angle of the $\gamma / \gamma^{\prime}$ interface versus life consumption ratio curve of Fig. 4 is very similar to that of the creep curve of Fig. 1. This clearly indicates that the accumulation of creep strain in Ni-based superalloys at high temperature and low stress occurs so as to increase the extent of the tilting of the $\gamma / \gamma^{\prime}$ interface from the (001) plane in the raft structure. The generality of the method developed by Epishin et al. [9] to characterize the microstructure evolution of Ni-based superalloys during creep deformation is thus confirmed. 
The increase in the tilt angle in the tertiary creep stage is obviously due to the increase in areas where the $\gamma / \gamma^{\prime}$ interface tilts significantly from the (001) plane. However, the tilt angle seems not to be homogeneously distributed but to be distributed heterogeneously at some particular tilt angles, as shown in Fig. 5. Fig. 5 shows an angular distribution profile of the FT spectrum obtained on (110) planes at the life consumption ratio of 0.95 . The profile is far from the Gaussian distribution but contains sub-peaks at angles away from that corresponding to the maximum peak by about $\pm 30^{\circ}$. Correspondingly, many $\gamma / \gamma^{\prime}$ interfaces with these angles are observed very frequently (Fig. 2(d)). At rupture, the tilt angle further increases, as shown in Fig. 2(e). We suspect that the mechanism through which the $\gamma / \gamma^{\prime}$ interface tilts from the (001) plane in the tertiary creep stage is different from that in the secondary creep stage. The reason why $\gamma / \gamma^{\prime}$ interfaces with particular tilt angles $\left( \pm 30^{\circ}\right)$ dominate in the tertiary creep stage has yet to be clarified.

\section{Conclusions}

1. The evolution of the raft structure can be characterized quantitatively by obtaining Fourier transformation spectra from SEM images of deformation microstructures. The result of analysis does not depend on crystallographic planes of SEM observations.

2. When judged from the similarity of Fig. 3 (the tilt angle of the $\gamma / \gamma^{\prime}$ interface versus life consumption ratio curve) and Fig. 4 (the creep curve), the tilt angle of the $\gamma / \gamma^{\prime}$ interface from the (001) plane increases with the deformation time as the accumulated creep strain does. In other words, the accumulation of creep strain in Ni-based superalloys at high temperature and low stress occurs so as to increase the extent of the tilting of the $\gamma / \gamma^{\prime}$ interface from the (001) plane in the raft structure.

3. In the tertiary creep stage, the tilt angle increases very rapidly to a very high level and many $\gamma / \gamma^{\prime}$ interfaces with particular tilt angles around $\pm 30^{\circ}$ are observed very frequently.

\section{Acknowledgments}

This work was supported by Grant-in-Aid for Scientific Research (A) (No.21246101 and No.21360337) from the Ministry of Education, Culture, Sports, Science and Technology (MEXT), Japan and in part by the Global COE (Center of Excellence) Program of International Center for Integrated Research and Advanced Education in Materials Science from the MEXT, Japan.

\section{Reference}

[1] J.K. Tien and S.M. Copley: Metall. Trans. Vol. 2(1971), p.215

[2] T.M. Pollock and A.S. Argon: Acta Metall. Mater. Vol. 42(1994), p.1859

[3] F.R.N. Nabarro: Metall. Trans. Vol. 27A(1996), p.513

[4] M. Veron, Y. Brechet and F. Louchet: Scr. Mater. Vol. 34(1996), p.1883

[5] J.Y. Buffiere and M. Ignat: Acta Metall. Mater. Vol. 43(1995), p.1791

[6] Y. Murata, R. Hashizume, A. Yoshinari, N. Aoki, M. Morinaga and Y. Fukui: Superalloys 2000(The Minerals, Metal and Materials Society, 2000), p.285 
[7] K. Tanaka, T. Kijikawa, T. Ichitsubo, M. Osawa, T. Yokokawa and H. Harada: Mater. Sci. Forum (2005), p.475

[8] K. Tnaka, T. Ichitsubo, K. Kishida, H. Inui and E. Matsubara: Acta Mater. Vol. 56(2008), p.3786

[9] A. Epishin, T. Link, P.D. Portella and U. Bruckner: Acta mater. Vol. 48(2000), p.4169

[10] R.C. Reed, N. Matan, D.C. Cox, M.A. Rist and C.M.F. Rae: Acta mater. Vol. 47(1999), p.3367

[11] T. Hino, T. Kobayashi, Y. Koizumi, H. Harada and T. Yamagata: Superalloys 2000(The Minerals, Metal and Materials Society, 2000), p.729

[12] P. Caron: Superalloys 2000(The Minerals, Metal and Materials Society, 2000), p.737

[13] J.X. Zhang, T. Murakumo, Y. Koizumi, T. Kobayashi, H. Harada and S. Masaki: Metall. Mater. Trans. Vol. 33A(2002), p.3741

[14] A.C. Yeh, C.M.F. Rae and S. Tin: Superalloys 2004(The Minerals, Metal and Materials Society, 2004), p.677 